\title{
Assessment of left ventricular myocardial work in Turner syndrome patients: insights from the novel non-invasive pressure- strain loop analysis method
}

\author{
Felix Sebastian Oberhoffer ${ }^{1}$, Hashim Abdul-Khaliq ${ }^{1}$, Anna-Maria Jung ${ }^{2}$, Michael Zemlin ${ }^{2}$, \\ Tilman R. Rohrer ${ }^{2}$, Mohamed Abd El Rahman ${ }^{1,3}$ \\ ${ }^{1}$ Department of Pediatric Cardiology, Saarland University Hospital, Homburg (Saar), Germany; ${ }^{2}$ Department of Pediatric Endocrinology, Saarland \\ University Hospital, Homburg (Saar), Germany; ${ }^{3}$ Department of Pediatrics and Pediatric Cardiology, Cairo University, Cairo, Egypt
}

Correspondence to: Hashim Abdul-Khaliq, MD. Kirrberger Straße, Geb. 9, 66421 Homburg (Saar), Germany. Email: hashim.abdul-khaliq@uks.eu.

\begin{abstract}
Background: Turner syndrome (TS), a relatively rare chromosomal disease, is associated with multiple cardiovascular risk factors that possibly lead to increased left ventricular afterload and functional impairment. The aim of this study was to investigate whether alterations in myocardial work and work efficiency can be found in TS patients through left ventricular pressure-strain loop analysis (PSL).

Methods: Thirty-eight patients with TS and 19 healthy, age-matched controls were recruited for this study. Global peak systolic strain (GLPS) and PSL of the left ventricle was assessed in study participants. TS patients whose history included coarctation of the aorta or prior cardiac surgery were excluded from GLPS and PSL analyses $(\mathrm{n}=5)$.

Results: Median age was 16.00 years in the TS group and 16.35 years in the control group $(\mathrm{P}=0.236)$. GLPS did not show significant differences between both groups $(\mathrm{P}=0.524)$. TS patients demonstrated, compared to controls, a significantly higher global myocardial work index (BSA) (mean \pm SD: 1,497 \pm 505 vs. $1,214 \pm 245 \mathrm{mmHg} \% / \mathrm{m}^{2} ; \mathrm{P}=0.027$ ). Heart rate was significantly increased in TS patients, compared to controls (mean \pm SD: $90.08 \pm 14.79$ vs. $73.95 \pm 15.05$ bpm; $\mathrm{P}<0.001$ ), and correlated significantly with global myocardial work index [body surface area (BSA)] within the TS cohort $(\mathrm{r}=0.558, \mathrm{P}=0.001)$.

Conclusions: TS patients showed signs of increased myocardial workload that were only detectable through the novel PSL analysis method and not through GLPS. Moreover, elevated resting heart rate was linked with increased myocardial workload in TS patients. Further studies will have to investigate whether TS patients may develop advanced left ventricular systolic dysfunction later in life.
\end{abstract}

Keywords: Echocardiography; myocardial work; pressure-strain loop (PSL); Turner syndrome (TS)

Submitted Jun 26, 2019. Accepted for publication Sep 23, 2019.

doi: 10.21037/qims.2019.09.19

View this article at: http://dx.doi.org/10.21037/qims.2019.09.19

\section{Introduction}

Turner syndrome (TS) is an $\mathrm{X}$-chromosomal disease that occurs approximately in one out of 2,500 to 3,000 female newborns (1).

Girls and women with TS have an increased cardiovascular morbidity (2): congenital heart defects such as the bicuspid aortic valve or the coarctation of the aorta are frequently seen among this cohort (3). TS is also associated with multiple cardiovascular and cardiometabolic risk factors such as diabetes, lipid anomalies, arterial hypertension and excess weight (4). In addition, arterial stiffness, a predictive cardiovascular risk marker (5), has been shown to be elevated in TS patients (6-9).

The accumulation of the above-mentioned risk factors, which may also promote the arterial stiffening process of the small and major vessels, is assumed to increase 
left ventricular afterload and thus impair left ventricular function. Studies could demonstrate that TS patients display lower left ventricular function and higher left ventricular mass $(10,11)$. However, in a recent study of our group, the previously described left ventricular dysfunction could not be confirmed through two-dimensional speckle tracking echocardiography (2DSTE) (6).

Recently, a new echocardiographic method has been introduced allowing the measurement of myocardial work and myocardial efficiency through left ventricular pressurestrain loops $(12,13)$.

The aim of this study was to investigate whether alterations in myocardial work and work efficiency can be found in TS patients through left ventricular pressurestrain loop analysis (PSL) in comparison to conventional left ventricular global strain assessment.

\section{Methods}

\section{Ethical statement}

This study was a retrospective analysis of prospectively collected data and was approved by the Ethics Committee of the Ärztekammer des Saarlandes (State Chamber of Physicians of the German federal state of Saarland), Faktoreistraße 4, 66111 Saarbrücken, Germany, on March 23rd, 2018; approval statement No. 07/18. This study conformed to the standards set by the latest revision of the Declaration of Helsinki. Prior written informed consent was obtained from all patients or the parents or legal guardians of patients under legal age.

\section{Study population}

Thirty-eight TS patients and 19 healthy, age-matched controls participated in this study. Study subjects were recruited between November 2016 and April 2018. TS patients examined for clinical routine inspection at the departments of pediatric cardiology and pediatric endocrinology of Saarland University Hospital were chosen. In addition, external TS patients were included in this study through collaboration with the German Turner Syndrome Association (Turner-Syndrom-Vereinigung Deutschland e.V.). The control group was composed of healthy, agematched volunteers and patients with accidental heart murmurs in whom cardiac pathologies were ruled out by echocardiography and electrocardiography. Body surface area $\left(\mathrm{BSA}, \mathrm{m}^{2}\right)$ was assessed according to Mosteller's formula (14). In study subjects $<18$ years of age, weight classification was evaluated according to body mass index (BMI, $\mathrm{kg} / \mathrm{m}^{2}$ ) percentiles established by Kromeyer-Hauschild et al. (15). In study participants $\geq 18$ years of age, normal weight was defined as BMI $<25 \mathrm{~kg} / \mathrm{m}^{2}$, overweight as BMI $\geq 25 \mathrm{~kg} / \mathrm{m}^{2}$ but $<30 \mathrm{~kg} / \mathrm{m}^{2}$ and obesity as BMI $\geq 30 \mathrm{~kg} / \mathrm{m}^{2}$.

\section{Echocardiography}

A 2.5-3.5 MHz phased array transducer with a Vivid 9 ultrasound system (General Electrics Healthcare, Fairfield, CT, USA) was applied for the entire echocardiographic examination.

\section{Left ventricular dimensions}

Left ventricular dimensions were obtained through M-Mode echocardiography in parasternal long axis view at the tip of the mitral valve. Left ventricular borders were evaluated at end-diastole (QRS complex in ECG) and end-systole (end of T-wave in ECG). The variables listed below were assessed: interventricular septum thickness at end-diastole (IVSd, cm), interventricular septum thickness at end-systole (IVSs, cm), left ventricular internal dimension at end-diastole (LVIDd, $\mathrm{cm}$ ), left ventricular internal dimension at end-systole (LVIDs, $\mathrm{cm})$, left ventricular posterior wall thickness at end-diastole (LVPWd, cm), left ventricular posterior wall thickness at endsystole (LVPWs, cm), left ventricular end-diastolic volume (EDV, $\mathrm{mL}$ ), left ventricular end-systolic volume (ESV, $\mathrm{mL}$ ), stroke volume (SV, $\mathrm{mL})$, ejection fraction (EF, \%), fractional shortening (FS, \%), left ventricular end-diastolic mass (LV Mass, g). "Z-scores of cardiac structures" were measured according to Pettersen et al. for IVSd, IVSs, LVIDd, LVIDs, LVPWd and LVPWs (16). EDV, ESV, SV and LV Mass were put in relation to BSA.

\section{End-systolic wall stress}

End-systolic wall stress (ESWS, $\times 10^{3}$ dynes $/ \mathrm{cm}^{2}$ ), which is considered to reflect left ventricular afterload, was calculated according to Wilson et al. using the following formula $(17,18)$ :

$$
E S W S=0.98 \times \frac{0.334 \times S B P \times L V I D s}{L V P W s} \times\left(1+\frac{L V P W s}{L V I D s}\right)-2
$$

TS patients with history of coarctation of the aorta or prior cardiac surgery were excluded from ESWS analysis.

\section{Left ventricular systolic deformation}

Left ventricular systolic deformation was evaluated 
through 2DSTE. Detailed information on methodological procedure of 2DSTE has been described in a recent publication of our group (6). In short: A constant frame rate of 60-90 fps was applied during echocardiographic examination of the left ventricle in apical two chamber view, apical three chamber view and apical four chamber view. We then utilized a separated workstation (EchoPAC PC version 202, General Electrics Healthcare) to analyse recorded loops offline. The endocardium of the left ventricle was marked precisely, and the region of interest was adapted to the myocardial thickness. Time of end-systole was represented by aortic valve closure. The software then generated a GLPS for the apical two chamber view (GLPS_A2C, \%), the apical three chamber view (GLPS_A3C, \%) and the apical four chamber view (GLPS_A4C, \%). In addition, an average (GLPS_AVG, \%) of the three assessed chamber views (GLPS_A2C, GLPS A3C, GLPS_A4C) was determined. TS patients with history of coarctation of the aorta or prior cardiac surgery were excluded from left ventricular 2DSTE analysis.

\section{Left ventricular myocardial work and efficiency}

To assess left ventricular myocardial work and efficiency, a non-invasive pressure-strain loop was generated (PSL). The procedure was executed offline through a semi-automatic software (EchoPAC PC version 202, General Electrics Healthcare). Brachial systolic blood pressure (SBP, mmHg) was measured simultaneously during echocardiographic examination. Left ventricular strain, which was required for PSL composition, was assessed through 2DSTE. Time of isovolumic and ejection phase was set manually by evaluating mitral and aortic valvular events through echocardiography in the apical three chamber view.

The left ventricular pressure curve generation was validated by Russell et al. through invasive cardiac catheterization. For the individual non-invasive PSL analysis the left ventricular pressure curve of reference is then adjusted by modifying its amplitude (SBP) and width (valvular events) (13).

The area within the PSL is considered as an index of global myocardial work ( $\left.\mathrm{mmHg}^{*} \%\right)$ (18). Global constructive work $\left(\mathrm{mmHg}^{*} \%\right)$ comprises myocardial work leading to myocardial shortening and thus to left ventricular ejection (18). Myocardial work that is not leading to left ventricular ejection is considered as global wasted work $\left(\mathrm{mmHg}^{*} \%\right)$ and includes dyssynchronous muscle fibres which are stretching during systole $(13,18)$.
Global ConstructiveWork

The ratio: $\overline{\text { Global ConstructiveWork+GlobalWasted Work }}$ is defined as global myocardial work efficiency (\%) (18). For better comparability of the TS and the control group, global myocardial work index, global constructive work and global wasted work were indexed to BSA $\left(\mathrm{mmHg}^{*} \% / \mathrm{m}^{2}\right)$. In addition, TS patients with history of coarctation of the aorta or prior cardiac surgery were excluded from PSL analysis. Figure 1 illustrates the non-invasive left ventricular PSL, myocardial work and myocardial efficiency.

\section{Pulse wave velocity}

Pulse wave velocity (PWV, m/s), a parameter to evaluate vascular stiffness, was evaluated non-invasively through an oscillometric blood pressure measuring device (MobilO-Graph, I.E.M. GmbH, Stolberg, Germany). Detailed information on methodological background has been described in a recent publication of our group (6). The Mobil-O-Graph has been validated through invasive catheter examinations (19). In addition, device specific PWV reference values exist for healthy pediatric and adult cohorts $(20,21)$. Cuff sizes were chosen according to the left upper arm circumference. The PWV analysis was only executed after the study participant had been resting for at least 5 to 10 minutes in a seated position.

TS patients with history of coarctation of the aorta or prior cardiac surgery were excluded from PWV analysis.

Figure 2 represents schematically the influence of PWV on central hemodynamics.

\section{Statistical analyses}

For statistical analyses SPSS 24 (Released 2016. IBM SPSS Statistics for Windows, Version 24.0. IBM Corp., Armonk, NY, USA) was used. The Kolmogorov-Smirnov test was utilized to evaluate normality of continuous variables. Data are presented as mean \pm SD or as median, minimum and maximum if not normally distributed. The independentsamples t-test was applied to compare continuous variables with normal distribution. Non-normally distributed continuous variables were compared using the MannWhitney $U$ test. For correlation analyses, the Pearson correlation was utilized for normally distributed variables or the Spearman correlation for non-normally distributed variables. A $\mathrm{P}$ value $<0.05$ was considered as statistically significant. 

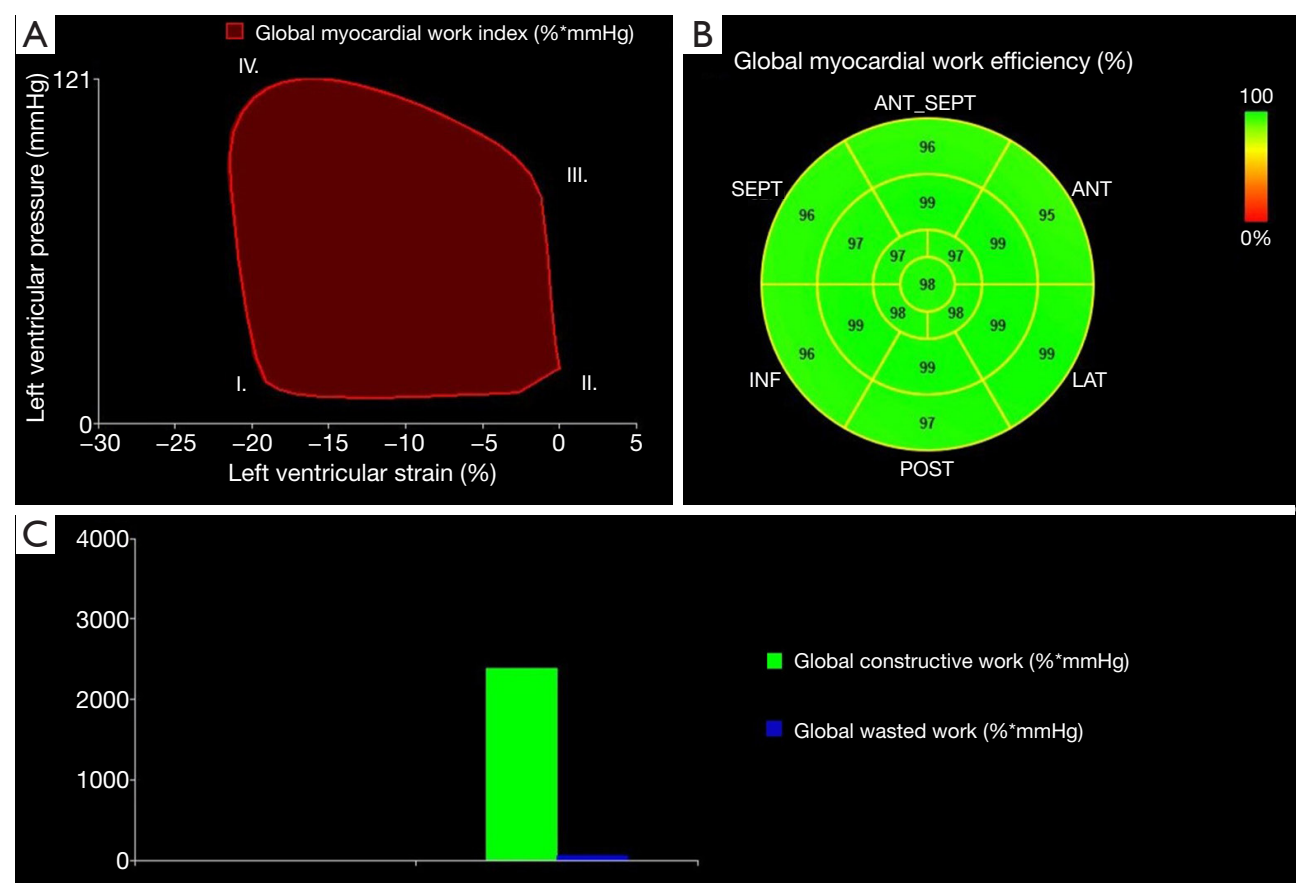

Figure 1 Non-invasive left ventricular pressure-strain loop, myocardial work and myocardial efficiency. (A) Illustration of the non-invasive left ventricular pressure-strain loop. The area within the pressure-strain loop represents the global myocardial work index (\% $\left.{ }^{*} \mathrm{mmHg}\right)$. I, mitral valve opening; II, mitral valve closure; III, aortic valve opening; IV, aortic valve closure; I-II, diastolic filling; II-III, isovolumic contraction; III-IV, ejection; IV-I, isovolumic relaxation. (B) Illustration of the global myocardial work efficiency (\%) for single left ventricular segments. (C) Illustration of the left ventricular global constructive- and global wasted work (\%*mmHg). ANT_SEPT, anteroseptal segment; SEPT, septal segment; ANT, anterior segment; INF, inferior segment; LAT, lateral segment; POST, posterior segment.
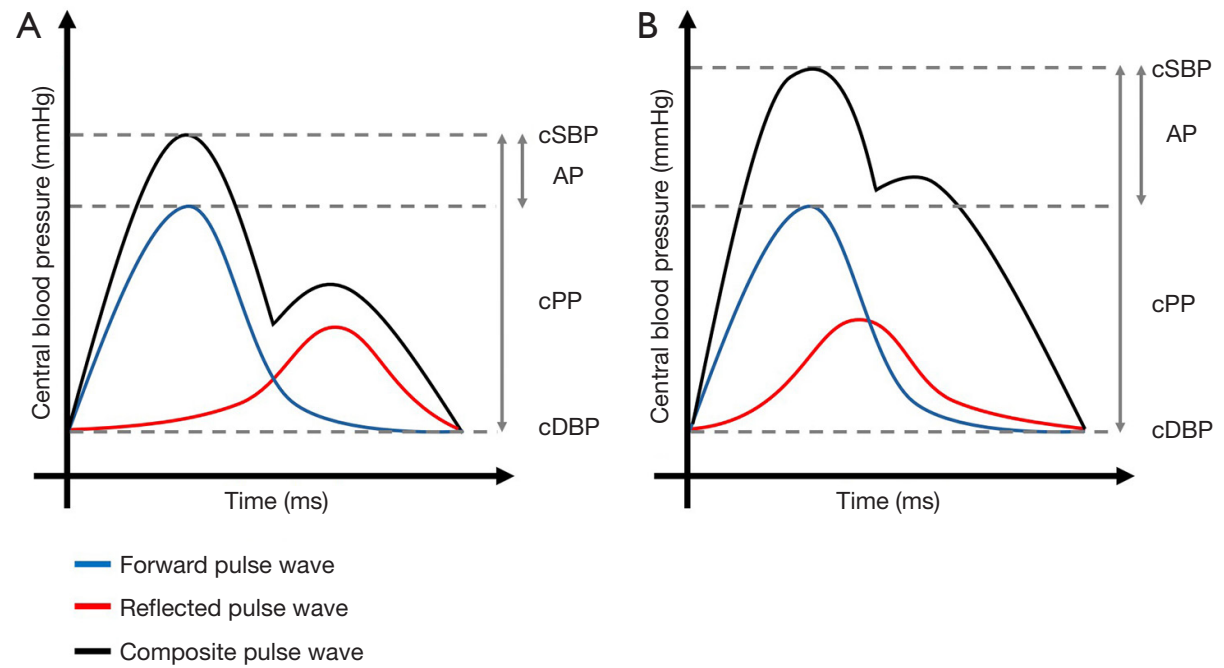

Figure 2 Schematic illustration of the influence of pulse wave velocity on central hemodynamics. (A) Healthy blood vessel. The periphery reflected pulse wave arrives at the end of the forward pulse wave due to a normal pulse wave velocity. The composite of both pulse waves leads to a small increase of the augmentation pressure and hence a small increase of the central systolic blood pressure and the left ventricular afterload. (B) Stiff blood vessel. An increase in a blood vessel's arterial stiffness results in a higher pulse wave velocity and therefore an earlier arrival of the periphery reflected pulse wave into the forward pulse wave. The composite of both pulse waves leads to a larger increase of the augmentation pressure and hence a larger increase of the central systolic blood pressure and the left ventricular afterload. cSBP, central systolic blood pressure; AP, augmentation pressure; cPP, central pulse pressure; cDBP, central diastolic blood pressure. 
Table 1 Patient characteristics

\begin{tabular}{lccc}
\hline Variables & Turner syndrome $(\mathrm{n}=38)$ & Controls $(\mathrm{n}=19)$ & $\mathrm{P}$ value \\
\hline Age (years) & $16.00(4.28 / 44.13)$ & $16.35(11.14 / 43.82)$ & 0.236 \\
Height $(\mathrm{cm})$ & $148.00(100.00 / 163.00)$ & $164.00(142.00 / 176.00)$ & $<0.001^{\star * *}$ \\
Weight $(\mathrm{kg})$ & $48.63 \pm 20.16$ & $54.69 \pm 9.07$ & 0.124 \\
BMI $\left(\mathrm{kg} / \mathrm{m}^{2}\right)$ & $22.98 \pm 6.27$ & $20.35 \pm 2.26$ & $0.025^{\star}$ \\
BSA $\left(\mathrm{m}^{2}\right)$ & $1.37 \pm 0.35$ & $1.57 \pm 0.16$ & $0.004^{\star *}$ \\
Heart rate $(\mathrm{bpm})$ & $90.08 \pm 14.79$ & $73.95 \pm 15.05$ & $<0.001^{\star * *}$ \\
SBP $(\mathrm{mmHg})$ & $119.45 \pm 15.51$ & $116.68 \pm 8.25$ & 0.384 \\
DBP $(\mathrm{mmHg})$ & $73.61 \pm 12.69$ & $69.84 \pm 9.41$ & 0.258 \\
\hline
\end{tabular}

Mean \pm standard deviation is used for normally distributed variables and median (minimum/maximum) for non-normally distributed variables; *, $\mathrm{P}<0.05$; ${ }^{* *}, \mathrm{P} \leq 0.01$; **, $\mathrm{P} \leq 0.001$. BMI, body mass index; BSA, body surface area; SBP, brachial systolic blood pressure; DBP, brachial diastolic blood pressure.

\section{Results}

\section{Patient characteristics}

The median age was 16.00 years for the TS group and 16.35 years for the control group. Both groups did not differ significantly in age. TS patients were, compared to controls, significantly smaller in height $(\mathrm{P}<0.001)$ and showed a significantly lower BSA $(\mathrm{P}=0.004)$. $\mathrm{BMI}(\mathrm{P}=0.025)$ and resting heart rate $(\mathrm{P}<0.001)$ were significantly increased in the TS group. No significant differences were assessed in weight, SBP and DBP between TS patients and control subjects. Detailed information on patient characteristics are summarized in Table 1.

\section{Karyotype and cardiovascular morbidity of TS patients}

Out of 38 TS patients, 21 (55.3\%) displayed a monosomy $\mathrm{X}, 12(31.6 \%)$ a mosaic form of TS, and $2(5.3 \%)$ structural chromosomal aberrations. Three (7.9\%) TS patients had diagnosis of "TS unspecified (Q. 96.9)".

Overall, $15(39.5 \%)$ TS patients presented with congenital heart disease. Ten $(26.3 \%)$ TS patients were diagnosed with bicuspid aortic valve and $2(5.3 \%)$ with monocuspid aortic valve. Five (13.2\%) TS patients had prior diagnosis of coarctation of the aorta. Four (10.5\%) TS patients displayed partial anomalous pulmonary venous drainage and $3(7.9 \%)$ showed dilatation of the ascending aorta. Four $(10.5 \%)$ TS patients underwent prior cardiac surgery. Of note, some TS individuals demonstrated multiple congenital heart defects.

Twenty-two (57.9\%) TS patients were classified as normal weight, $9(23.7 \%)$ as overweight and $7(18.4 \%)$ as obese. Five $(13.2 \%)$ TS patients had arterial hypertension and 3 (7.9\%) showed carbohydrate metabolism disorders. Results on karyotype and cardiovascular morbidity of TS patients are summarised in Table 2.

\section{Echocardiography}

\section{Left ventricular dimensions}

Compared to controls, TS subjects displayed significantly higher values for IVSd $\mathrm{Z}$ score $(\mathrm{P}=0.030)$. Detailed information on left ventricular dimension is summarized in Table 3 for both groups.

\section{End-systolic wall stress}

No significant difference was assessed in ESWS between TS patients $(n=33)$ and controls $(n=19)$ in regard to different parameters indicating left ventricular wall stress (median, minimum/maximum: $125.77 \times 10^{3}, 91.22 \times 10^{3} / 187.96 \times 10^{3}$ vs. $122.98 \times 10^{3}, 103.07 \times 10^{3} / 156.10 \times 10^{3}$ dynes $/ \mathrm{cm}^{2}$; $\mathrm{P}=0.403)$. However, within the TS group, overweight and obese TS subjects $(n=13)$ showed, compared to normal weight TS subjects $(n=20)$, significantly higher values in ESWS (median, minimum/maximum: $131.43 \times 10^{3}, 111.16 \times 10^{3} / 187.96 \times 10^{3}$ vs. $123.68 \times 10^{3}$, $91.22 \times 10^{3} / 172.40 \times 10^{3}$ dynes $\left./ \mathrm{cm}^{2} ; \mathrm{P}=0.006\right)$. Figure 3 visualizes differences in ESWS between normal weight and overweight/obese TS subjects.

\section{Left ventricular systolic deformation}

No significant difference in GLPS_A2C, GLPS_A3C, 
Table 2 Karyotype and cardiovascular morbidity of Turner syndrome patients

\begin{tabular}{lc}
\hline Variables & Turner syndrome $(\mathrm{n}=38)$ \\
\hline Karyotype, $\mathrm{n}(\%)$ & $21(55.3)$ \\
$45, \mathrm{X0}$ & $12(31.6)$ \\
Mosaic form & $2(5.3)$ \\
Structural chromosomal aberration & $3(7.9)$ \\
Unspecified (Q. 96.9) & $15(39.5)$ \\
Congenital heart disease, $\mathrm{n}(\%)$ & $10(26.3)$ \\
Bicuspid aortic valve & $2(5.3)$ \\
Monocuspid aortic valve & $5(13.2)$ \\
Coarctation of the aorta & $4(10.5)$ \\
Partial anomalous pulmonary venous & \\
drainage & $3(7.9)$ \\
Dilatation of the ascending aorta & $4(10.5)$ \\
Cardiac surgery, $\mathrm{n}(\%)$ & \\
Weight classification, $\mathrm{n}$ (\%) & $22(57.9)$ \\
Normal weight & $9(23.7)$ \\
Overweight & $7(18.4)$ \\
Obese & $5(13.2)$ \\
Arterial hypertension, $\mathrm{n}$ (\%) & $3(7.9)$ \\
Carbohydrate metabolism disorders, & \\
\hline
\end{tabular}

GLPS_A4C and the subsequently calculated GLPS_AVG was found between the TS and the healthy control group. Detailed data on measured left ventricular deformation is summarized in Table 4 for both groups.

\section{Left ventricular myocardial work and efficiency}

Using the novel PSL analysis method, TS patients achieved, compared to controls, significantly higher values in global myocardial work index $(\mathrm{BSA})(\mathrm{P}=0.027)$ and global constructive work (BSA) $(\mathrm{P}=0.011)$. Both groups did not show significant differences in global wasted work (BSA) and global myocardial work efficiency. Within the TS cohort, heart rate correlated significantly with global myocardial work index (BSA) $(\mathrm{r}=0.558, \mathrm{P}=0.001)$ and global constructive work (BSA) $(r=0.445, P=0.009)$. Detailed results on left ventricular myocardial work and efficiency are summarized in Table 4.

\section{Pulse wave velocity}

Arterial stiffness was non-invasively assessed through PWV analysis as described in the methods. Of note, PWV analysis was not executed in three control subjects. PWV did not differ significantly between TS patients $(\mathrm{n}=33)$ and controls ( $\mathrm{n}=16)$ (median, minimum/maximum: 4.80, 3.80/6.90 vs. $4.75,3.90 / 6.50 \mathrm{~m} / \mathrm{s} ; \mathrm{P}=0.661)$. Overweight and obese TS

Table 3 Left ventricular dimensions in Turner syndrome patients and controls

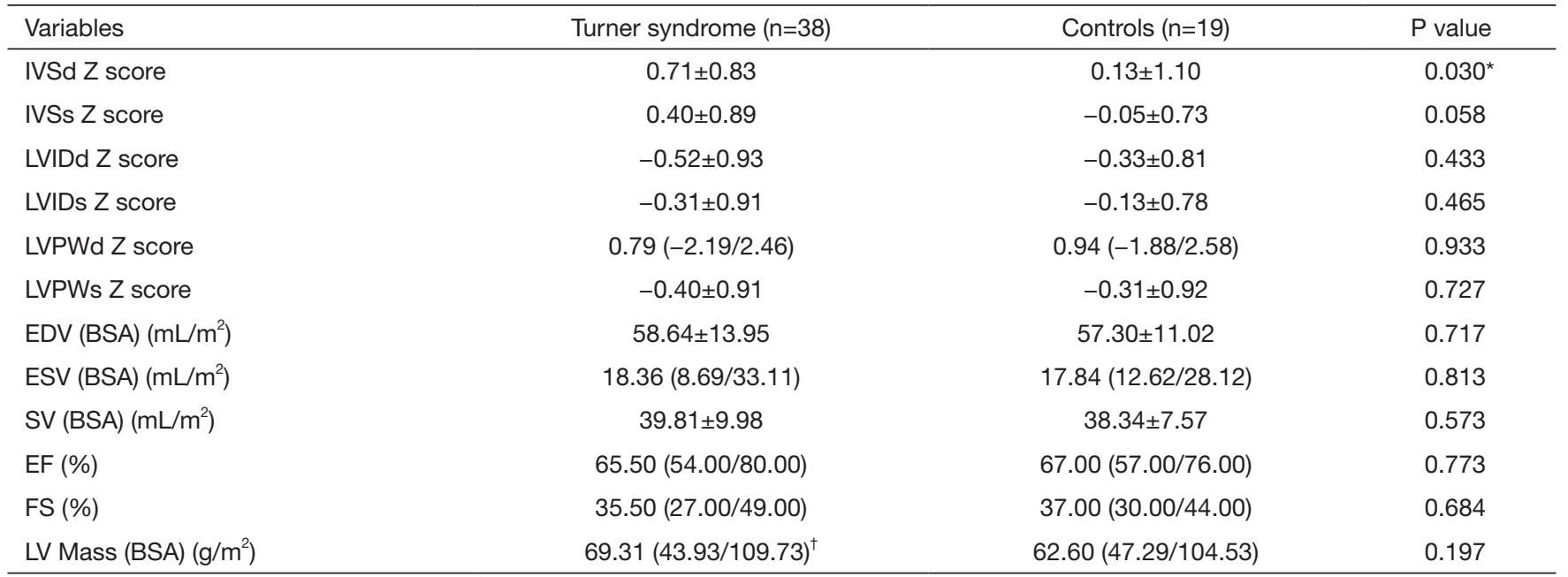

Mean \pm standard deviation is used for normally distributed variables and median (minimum/maximum) for non-normally distributed variables; * $\mathrm{P}<0.05 ;{ }^{\dagger}$, results include 37 patients with Turner syndrome. IVSd, interventricular septum thickness at end-diastole; IVSs, interventricular septum thickness at end-systole; LVIDd, left ventricular internal dimension at end-diastole; LVIDs, left ventricular internal dimension at end-systole; LVPWd, left ventricular posterior wall thickness at end-diastole; LVPWs, left ventricular posterior wall thickness at end-systole; BSA, body surface area; EDV, left ventricular end-diastolic volume; ESV, left ventricular end-systolic volume; SV, stroke volume; EF, ejection fraction; FS, fractional shortening, LV mass, left ventricular end-diastolic mass. 
patients $(n=13)$ tended to have, compared to normal weight TS subjects $(n=20)$, higher values for PWV (mean \pm SD: $5.06 \pm 0.41$ vs. $4.75 \pm 0.73 \mathrm{~m} / \mathrm{s} ; \mathrm{P}=0.174)$. Within the TS cohort, PWV correlated significantly with ESWS ( $\mathrm{r}=0.582$, $\mathrm{P}<0.001)$.

\section{Discussion}

\section{Increased myocardial work in TS patients}

To the best of our knowledge, this is the first study that applies the novel PSL analysis method in patients with TS. Compared to controls, TS patients demonstrated a significantly higher myocardial workload according

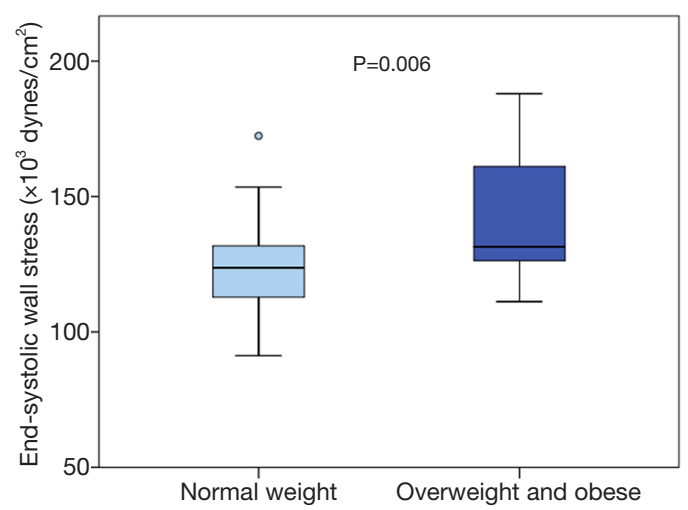

Figure 3 End-systolic wall stress $\left(\times 10^{3}\right.$ dynes $\left./ \mathrm{cm}^{2}\right)$ in normal weight and overweight/obese patients with Turner syndrome. to global myocardial work index (BSA) and global constructive work (BSA) which were assessed through PSL analysis.

The elevated myocardial work, observed in the TS cohort, might be primarily explained by the cumulative overall cardiovascular morbidity linked with TS (2-4,6-8) which leads to increased left ventricular afterload and possible functional myocardial impairment.

In a recent publication of our group, we were able to demonstrate signs of significantly increased arterial stiffness in TS patients by means of abdominal aortic 2DST (6). In this study, we evaluated arterial stiffness only through oscillometric PWV which is a validated non-invasive method (19). However, no significant differences in arterial stiffness were found between both groups by means of the oscillometric PWV method used. This may be explained by the relatively young age of the population studied. In addition, oscillometric PWV analysis may not be sensitive enough to detect early manifestations of arterial stiffening in young age.

Previous studies have shown that excess weight negatively affects arterial stiffness $(22,23)$. In this study, PWV tended to be higher in overweight and obese TS subjects, compared to normal weight TS subjects. In addition, we were able to demonstrate that overweight and obese TS subjects showed significantly higher ESWS when compared to normal weight TS subjects (Figure 3). Interestingly, PWV correlated significantly with ESWS within the TS cohort. These results might suggest that

Table 4 Left ventricular deformation, myocardial work and myocardial efficiency in Turner syndrome patients and controls

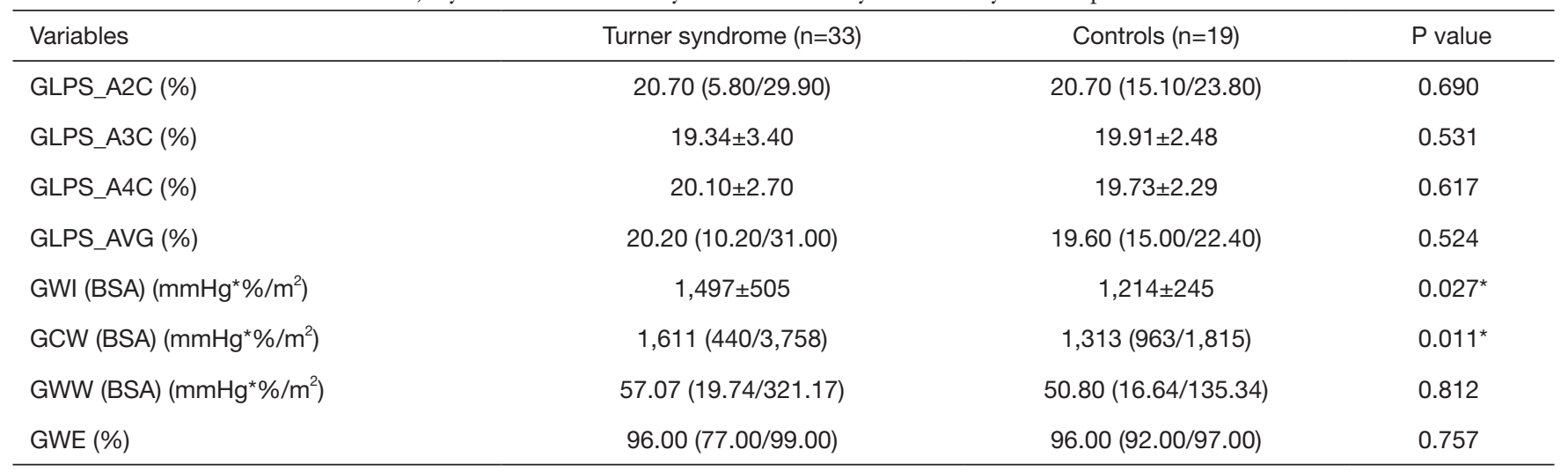

Mean \pm standard deviation is used for normally distributed variables and median (minimum/maximum) for non-normally distributed variables; *, P<0.05; GLPS_A2C, global peak systolic strain apical two chamber view; GLPS_A3C, global peak systolic strain apical three chamber view; GLPS_A4C, global peak systolic strain apical four chamber view; GLPS_AVG, averaged global peak systolic strain; GWI, global myocardial work index; GCW, global constructive work; GWW, global wasted work; GWE, global myocardial work efficiency; BSA, body surface area. 
left ventricular afterload is higher in overweight and obese TS patients secondary to an increased arterial stiffness. We therefore assume that TS patients with excess weight display an even higher cardiovascular risk when compared to normal weight TS patients. Consequently, the promotion of a healthy lifestyle is crucial in cardiovascular risk management of TS.

\section{Effects of heart rate on myocardial work in TS patients} TS is linked with an elevated resting heart rate, presumably caused by a sympathetic dysregulation $(10,24,25)$. The examined TS subjects in this study demonstrated, compared to healthy, age-matched controls, significantly increased values for resting heart rate. Interestingly, higher values for resting heart rate were closely associated with increased myocardial work within the TS group. Opdahl et al. demonstrated that an increase in resting heart rate is related to left ventricular dysfunction and an elevated risk of developing heart failure (26). A permanent increase in resting heart rate is accompanied by higher myocardial oxygen demand which possibly promotes the process of left ventricular remodelling (26). These results might suggest the use of resting heart rate as risk stratification for left ventricular dysfunction within the TS cohort possibly leading to an earlier therapeutic pharmacological intervention in at-risk patients to prevent further cardiovascular morbidities. Further studies are required to investigate whether heart rate lowering medications (e.g., beta-blockers or funny channel inhibitors) might reduce myocardial work in TS patients.

\section{GLPS vs. PSL}

Interestingly, left ventricular GLPS was not significantly altered between TS patients and healthy controls. These results might be due to the fact that GLPS is a load dependent parameter (18). Through PSL construction, however, left ventricular afterload is considered in the left ventricular function analysis (18). In patients with increased left ventricular afterload, the myocardium must apply more work to maintain left ventricular systolic function, which is conventionally measured by EF and GLPS (18). In this case, the global myocardial work index would be increased while the GLPS would still seem to be unchanged (18). In the long-term, however, a permanently raised global myocardial work index might result in left ventricular remodelling and left ventricular dysfunction (18). According to our preliminary results, myocardial work and efficiency parameters assessed by means of the recently developed
PSL analysis method may help to identify patients at risk of left ventricular remodelling and left ventricular systolic dysfunction even before abnormalities in GLPS can be detected.

Myocardial efficiency measured by global myocardial work efficiency did not differ significantly between TS patients and controls. The global myocardial work efficiency is mainly reduced by a relative increase in global wasted work compared to global constructive work. Global wasted work, however, has been shown to be primarily elevated in patients displaying heart rhythm disorders (e.g., left bundle branch block) leading to dyssynchronous movements of single myocardial segments (13). In this study, no significant differences in global wasted work (BSA) and global myocardial work efficiency were assessed between TS patients and controls. Since none of the examined TS patients presented with significant arrythmia, left bundle branch block or advanced heart failure, these results seem to be plausible. However, TS has been described to be associated with heart rhythm disorders (27-29). Further studies will have to investigate whether TS patients displaying heart rhythm disorders also show abnormalities in global wasted work and hence global myocardial work efficiency.

\section{Limitations}

\section{Study population}

This study was a cross-sectional single-center study. Taking into consideration the low prevalence of TS in the general population, the TS sample size applied in this study was relatively high. However, due to the heterogenic cardiovascular morbidity of the TS cohort studied, the effects of single cardiovascular risk factors on left ventricular function are still speculative. By establishing longitudinal multi-center studies, that include larger TS cohorts, the cardiovascular pathophysiology of TS could be better evaluated.

Moreover, the examined TS cohort consisted of relatively young study participants. It therefore remains unclear whether TS patients develop more advanced left ventricular systolic dysfunction later in life and whether PSL analysis might be beneficial for risk stratification.

\section{PSL and speckle tracking echocardiography}

PSL construction is dependent on visual determination of valve opening and closure time in the apical three chamber view. In the present study, we used a relatively low frame 
rate of 60-90 fps. To adequately determine short time intervals (e.g., isovolumetric contraction period), higher frame rates might be required. Especially in patients with elevated heart rates, the precise visual assessment of short time intervals through valve motion is difficult. An increase in frame rate, however, might result in less accurate tracking of myocardial speckles and therefore in lower quality strain assessment and calculation. The determination of short time intervals through Doppler echocardiography might be a possible solution to assess isovolumetric time intervals more precisely. In addition, the accuracy of 2DSTE is dependent on the sonographic window and on heart rate. Excess weight, which often leads to reduced image quality, as well as an elevated heart rate, impair myocardial speckle tracking which might result in imprecise strain values.

\section{Pulse wave velocity}

In this study, PWV was assessed non-invasively through the validated oscillometric Mobil-O-Graph device. In comparison to other similar non-invasive PWV measuring devices, the Mobil-O-Graph is reported to underestimate PWV values (30). Therefore, the PWV values received in this study might be device specific and not entirely applicable with other non-invasive PWV measuring devices (30).

\section{Conclusions}

In this study, TS patients showed signs of increased myocardial workload that were only detectable by means of the novel PSL analysis method and not through conventional GLPS. In addition, elevated resting heart rate was linked with increased myocardial workload in TS subjects. The changes in myocardial work might be interpreted as an adaptative remodelling of the left ventricular myocardium in response to an abnormal left ventricular afterload and to structural as well as functional vascular impairments in TS patients.

Further studies will have to investigate whether TS patients develop advanced left ventricular systolic dysfunction later in life and whether PSL analysis might be beneficial for risk stratification in these patients. Moreover, the possible influence of interventional therapeutic medications on lowering heart rate and myocardial work in TS should be evaluated in future studies.

\section{Acknowledgments}

We would like to thank all study participants and the
German Turner Syndrome Association (Turner-SyndromVereinigung Deutschland e.V.) for the interest in our study. Funding: This study was supported by the Competence Network for Congenital Heart Defects, which received funding from the Federal Ministry of Education and Research, Grant Number 01GI0601 [2014], and the German Centre for Cardiovascular Research, Grant Number 81X2800112 [2015].

\section{Footnote}

Conflicts of Interest: The authors have no conflicts of interest to declare.

Ethical Statement: This study was a retrospective analysis of prospectively collected data and was approved by the Ethics Committee of the Ärztekammer des Saarlandes (State Chamber of Physicians of the German federal state of Saarland), Faktoreistraße 4, 66111 Saarbrücken, Germany, on March 23rd, 2018; approval statement No. 07/18. This study conformed to the standards set by the latest revision of the Declaration of Helsinki. Prior written informed consent was obtained from all patients or the parents or legal guardians of patients under legal age.

\section{References}

1. Sybert VP, McCauley E. Turner's syndrome. N Engl J Med 2004;351:1227-38.

2. Gravholt CH, Andersen NH, Conway GS, Dekkers OM, Geffner ME, Klein KO, Lin AE, Mauras N, Quigley CA, Rubin K, Sandberg DE, Sas TCJ, Silberbach M, Söderström-Anttila V, Stochholm K, van Alfenvan derVelden JA, Woelfle J, Backeljauw PF. Clinical practice guidelines for the care of girls and women with Turner syndrome: proceedings from the 2016 Cincinnati International Turner Syndrome Meeting. Eur J Endocrinol 2017;177:G1-70.

3. Mazzanti L, Cacciari E. Congenital heart disease in patients with Turner's syndrome. The Journal of Pediatrics 1998;133:688-92

4. Mavinkurve M, O'Gorman CS. Cardiometabolic and vascular risks in young and adolescent girls with Turner syndrome. BBA Clin 2015;3:304-9.

5. Mitchell GF, Hwang SJ, Vasan RS, Larson MG, Pencina MJ, Hamburg NM, Vita JA, Levy D, Benjamin EJ. Arterial stiffness and cardiovascular events: the Framingham Heart Study. Circulation 2010;121:505-11. 
6. Oberhoffer FS, Abdul-Khaliq H, Jung AM, Rohrer TR, Abd El Rahman M. Two-dimensional speckle tracking of the abdominal aorta: a novel approach to evaluate arterial stiffness in patients with Turner syndrome. Cardiovasc Diagn Ther 2019;9:S228-37.

7. An HS, Baek JS, Kim GB, Lee YA, Song MK, Kwon BS, Bae EJ, Noh CI. Impaired Vascular Function of the Aorta in Adolescents with Turner Syndrome. Pediatr Cardiol 2017;38:20-6.

8. Uçar A, Öz F, Baş F, Oflaz H, Nişli K, Tuğrul M, Yetim A, Darendeliler F, Saka N, Poyrazoğlu Ş, Bundak R. Increased arterial stiffness in young normotensive patients with Turner syndrome: associations with vascular biomarkers. Clin Endocrinol (Oxf) 2015;82:719-27.

9. De Groote K, Devos D, Van Herck K, De Wolf D, Van der Straaten S, Rietzschel E, Raes A, Vandekerckhove K, Panzer J, De Wilde H, De Schepper J. Increased aortic stiffness in prepubertal girls with Turner syndrome. J Cardiol 2017;69:201-7.

10. Andersen NH, Hjerrild BE, Sørensen K, Pedersen EM, Stochholm K, Gormsen LC, Hørlyck A, Christiansen JS, Gravholt CH. Subclinical left ventricular dysfunction in normotensive women with Turner's syndrome. Heart 2006;92:1516-7.

11. Sozen AB, Cefle K, Kudat H, Ozturk S, Oflaz H, Akkaya V, Palanduz S, Demirel S, Ozcan M, Goren $\mathrm{T}$, Guven O. Left ventricular thickness is increased in nonhypertensive Turner's syndrome. Echocardiography 2009;26:943-9.

12. Russell K, Eriksen M, Aaberge L, Wilhelmsen N, Skulstad H, Remme EW, Haugaa KH, Opdahl A, Fjeld JG, Gjesdal O, Edvardsen T, Smiseth OA. A novel clinical method for quantification of regional left ventricular pressure-strain loop area: a non-invasive index of myocardial work. Eur Heart J 2012;33:724-33.

13. Russell K, Eriksen M, Aaberge L, Wilhelmsen N, Skulstad H, Gjesdal O, Edvardsen T, Smiseth OA. Assessment of wasted myocardial work: a novel method to quantify energy loss due to uncoordinated left ventricular contractions. Am J Physiol Heart Circ Physiol 2013;305:H996-1003.

14. Mosteller RD. Simplified calculation of body-surface area. N Engl J Med 1987;317:1098.

15. Kromeyer-Hauschild K, Wabitsch M, Kunze D, Geller F, Geiß HC, Hesse V, Hippel A von, Jaeger U, Johnsen D, Korte W, Menner K, Müller G, Müller JM, NiemannPilatus A, Remer T, Schaefer F, Wittchen HU, Zabransky S, Zellner K, Ziegler A, Hebebrand J. Perzentile für den Body-mass-Index für das Kindes- und Jugendalter unter
Heranziehung verschiedener deutscher Stichproben. Monatsschr Kinderheilkd 2001;149:807-18.

16. Pettersen MD, Du W, Skeens ME, Humes RA. Regression equations for calculation of $\mathrm{z}$ scores of cardiac structures in a large cohort of healthy infants, children, and adolescents: an echocardiographic study. J Am Soc Echocardiogr 2008;21:922-34.

17. Wilson JR, Reichek N, Hirshfeld J, Claude A, Keller RN. Noninvasive assessment of load reduction in patients with asymptomatic aortic regurgitation. Am J Med 1980;68:664-74.

18. Chan J, Edwards NFA, Khandheria BK, Shiino K, Sabapathy S, Anderson B, Chamberlain R, Scalia GM. A new approach to assess myocardial work by non-invasive left ventricular pressure-strain relations in hypertension and dilated cardiomyopathy. Eur Heart J Cardiovasc Imaging 2019;20:31-9.

19. Hametner B, Wassertheurer S, Kropf J, Mayer C, Eber B, Weber T. Oscillometric estimation of aortic pulse wave velocity: comparison with intra-aortic catheter measurements. Blood Press Monit 2013;18:173-6.

20. Elmenhorst J, Hulpke-Wette M, Barta C, Dalla Pozza R, Springer S, Oberhoffer R. Percentiles for central blood pressure and pulse wave velocity in children and adolescents recorded with an oscillometric device. Atherosclerosis 2015;238:9-16.

21. Aristizábal-Ocampo D, Espíndola-Fernández D, GalloVillegas J. Pulse wave velocity reference values in 3,160 adults referred to a hypertension clinic for 24hour ambulatory blood pressure monitoring. Clin Exp Hypertens 2018. [Epub ahead of print]. doi: 10.1080/10641963.2018.1545853.

22. Cote AT, Phillips AA, Harris KC, Sandor GGS, Panagiotopoulos C, Devlin AM. Obesity and arterial stiffness in children: systematic review and meta-analysis. Arterioscler Thromb Vasc Biol 2015;35:1038-44.

23. Safar ME, Czernichow S, Blacher J. Obesity, arterial stiffness, and cardiovascular risk. J Am Soc Nephrol 2006;17:S109-11.

24. Gravholt $\mathrm{CH}$. Turner syndrome and the heart: cardiovascular complications and treatment strategies. Am J Cardiovasc Drugs 2002;2:401-13.

25. Zuckerman-Levin N, Zinder O, Greenberg A, Levin M, Jacob G, Hochberg Z'e. Physiological and catecholamine response to sympathetic stimulation in turner syndrome. Clin Endocrinol (Oxf) 2006;64:410-5.

26. Opdahl A, Ambale Venkatesh B, Fernandes VRS, Wu CO, Nasir K, Choi E-Y, Almeida ALC, Rosen B, Carvalho 
B, Edvardsen T, Bluemke DA, Lima JAC. Resting heart rate as predictor for left ventricular dysfunction and heart failure: MESA (Multi-Ethnic Study of Atherosclerosis). J Am Coll Cardiol 2014;63:1182-9.

27. Bondy CA, Van PL, Bakalov VK, Sachdev V, Malone CA, Ho VB, Rosing DR. Prolongation of the cardiac QTc interval in Turner syndrome. Medicine (Baltimore) 2006;85:75-81.

28. Sozen AB, Cefle K, Kudat H, Ozturk S, Oflaz H, Pamukcu B, Akkaya V, Isguven P, Palanduz S, Ozcan M, Goren $\mathrm{T}$, Guven O. Atrial and ventricular arryhthmogenic

Cite this article as: Oberhoffer FS, Abdul-Khaliq H, Jung AM, Zemlin M, Rohrer TR, Abd El Rahman M. Assessment of left ventricular myocardial work in Turner syndrome patients: insights from the novel non-invasive pressure-strain loop analysis method. Quant Imaging Med Surg 2020;10(1):15-25. doi: 10.21037/qims.2019.09.19 potential in Turner Syndrome. Pacing Clin Electrophysiol 2008;31:1140-5.

29. Bondy CA, Ceniceros I, Van PL, Bakalov VK, Rosing DR. Prolonged rate-corrected QT interval and other electrocardiogram abnormalities in girls with Turner syndrome. Pediatrics 2006;118:e1220-5.

30. Lithovius R, Gordin D, Forsblom C, Saraheimo M, Harjutsalo V, Groop PH. Ambulatory blood pressure and arterial stiffness in individuals with type 1 diabetes. Diabetologia 2018;61:1935-45. 